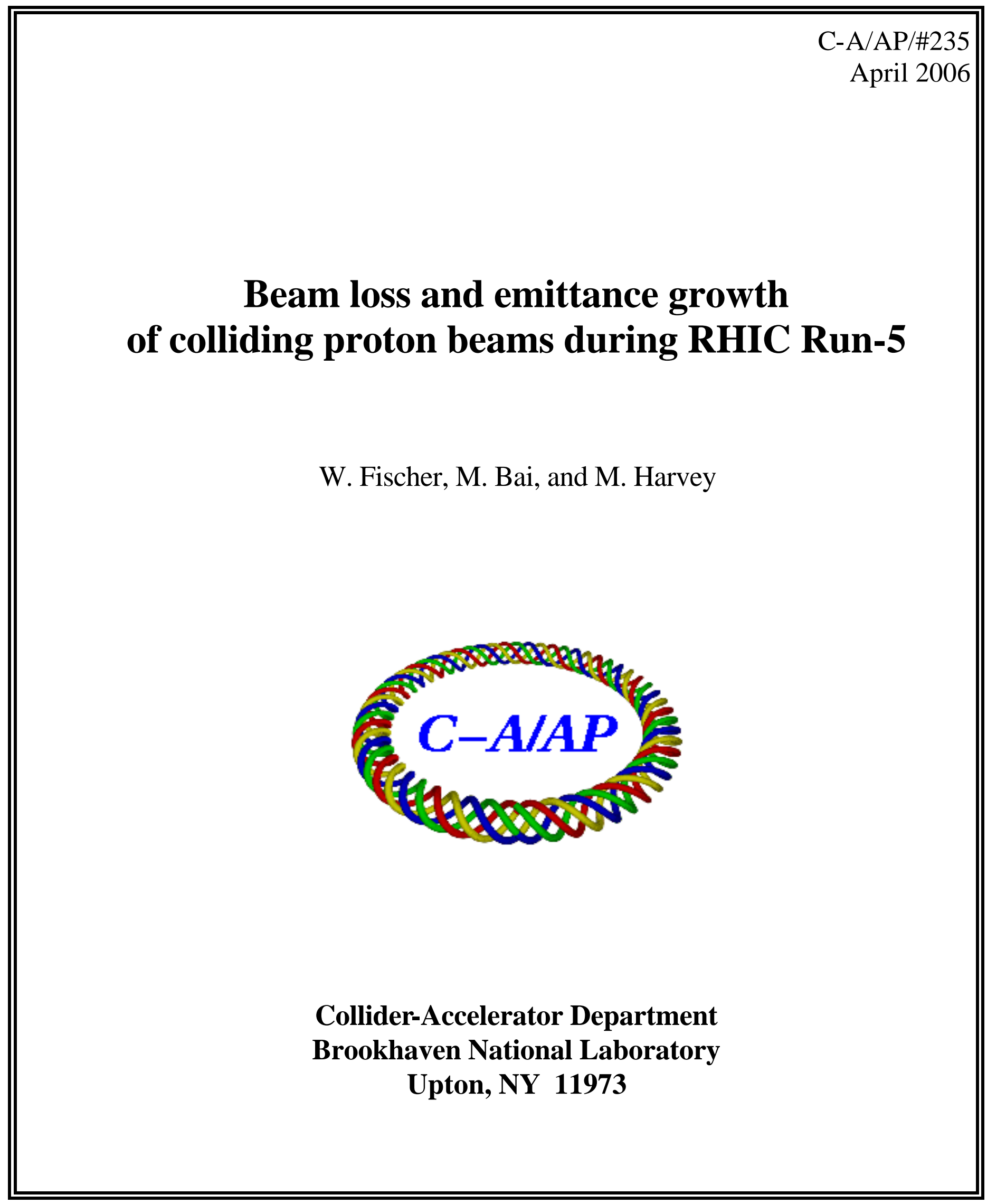


BNL C-A/AP/235

\title{
Beam loss and emittance growth of colliding proton beams during RHIC Run-5
}

\author{
W. Fischer, M. Bai, and M. Harvey
}

April 6, 2006

\begin{abstract}
The beam and luminosity lifetimes during the Run-5 polarized proton operation showed large variation from store to store. We report observed lifetimes and lifetime distributions. We calculate beam lifetimes and emittance growth due to luminosity, rest gas scattering, intrabeam scattering, and beam-beam elastic scattering for Run-5 parameters, the Enhanced Luminosity parameters, and RHIC II.
\end{abstract}

\section{Introduction}

During the polarized proton Run-5 the beam and luminosity lifetimes showed large variations. Fig. 1 shows two stores with rather different luminosities, beam and luminosity lifetimes, and background signals. During the run the cause for the lifetime variations could not be conclusively identified. Below we summarize the beam and luminosity observations and compare them with beam lifetime and emittance growth calculations. We consider beam losses due to luminosity and rest gas scattering, and emittance growth due to intrabeam scattering, elastic rest gas scattering, and beam-beam elastic scattering. Tab. 1 lists beam parameters for the Run-5 polarized proton operation. For the Run-5 analysis we restrict ourselves to stores at $100 \mathrm{GeV}$ beam energy.

Table 1: Beam parameters at the beginning of the store during polarized proton operation in Run-5.

\begin{tabular}{lcc}
\hline \hline quantity & unit & value \\
\hline proton energy & $\mathrm{GeV}$ & 100.0 \\
relativistic $\gamma$ & $\ldots$ & 106.6 \\
revolution time $T$ & $\mu \mathrm{s}$ & 12.8 \\
bunches per beam $N$ & $\ldots$ & $56-111$ \\
bunch intensity $N_{b}$ & $10^{11}$ & $0.39-1.05$ \\
normalized emittances $\epsilon_{n}(95 \%)$ & $\mathrm{mm} \mathrm{mrad}$ & $12-43$ \\
transverse tunes $\left(Q_{x}, Q_{y}\right)$, & $\ldots$ & $(28.68,29.69)$ \\
rf gap voltage & $\mathrm{kV}$ & 300 \\
rms bunch length & $\mathrm{m}$ & $0.5-1.2$ \\
number of head-on bb interaction $n_{I P}$ & $\ldots$ & 3 \\
initial beam-beam parameter $\xi / \mathrm{IP}$ & $\ldots$ & $0.001-0.005$ \\
\hline \hline
\end{tabular}



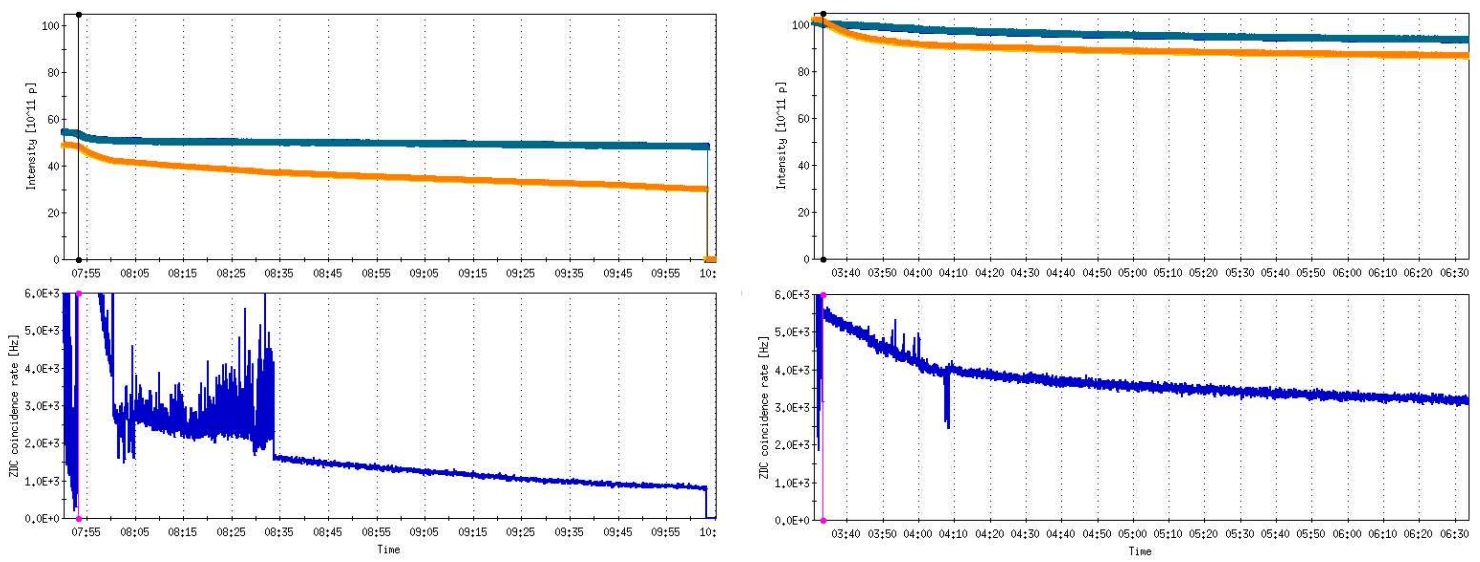

Figure 1: Beam intensities and PHENIX luminosity signal of two stores. Left, fill 7233, with moderate beam intensity in 58 bunches shows a poor Yellow beam lifetime, low initial luminosity, and a large background signal in the early part of the store. Right, fill 7327, has larger intensities in 106 bunches, and good beam and luminosity lifetimes. A change in the luminosity lifetime is visible after about half an hour, coinciding with a change in the Yellow beam lifetime.

We will use for the normalized transverse emittance

$$
\epsilon_{n}=(\beta \gamma) \frac{6 \sigma^{2}}{\beta}
$$

where $(\beta \gamma)$ are the relativistic factors, $\sigma$ the transverse $\mathrm{rms}$ beam size, and $\beta$ the lattice function. We assume the same emittance for both the horizontal and vertical plane, which is likely due to operation close to the main coupling resonance $Q_{x}=Q_{y}$. We also assume the same emittances for both the Blue and Yellow ring, a condition usually reached due to the beam-beam interaction.

The beam lifetime $\tau_{N_{b}}$ is defined through

$$
\frac{1}{\tau_{N_{b}}}=-\frac{1}{N_{b}} \frac{d N_{b}}{d t}
$$

where $N_{b}$ is the bunch intensity. The emittance growth time and luminosity lifetimes are defined through

$$
\frac{1}{\tau_{\epsilon_{n}}}=\frac{1}{\epsilon_{n}} \frac{d \epsilon_{n}}{d t} \text { and } \quad \frac{1}{\tau_{\mathcal{L}}}=-\frac{1}{\mathcal{L}} \frac{d \mathcal{L}}{d t}
$$

respectively, where, for round beams, the time dependent luminosity $\mathcal{L}(t)$ is

$$
\mathcal{L}(t)=\frac{3}{2 \pi}(\beta \gamma) \frac{f_{r e v} N}{\beta^{*}} \frac{N_{b, B}(t) N_{b, Y}(t)}{\epsilon_{n}(t)}
$$

$N$ is the number of bunch collisions per turn, $f_{\text {rev }}$ the revolution frequency, and $\beta^{*}$ the lattice function at the interaction point (IP), assumed to be the same for the horizontal 
and vertical plane. $N_{b, B}$ and $N_{b, Y}$ denote the Blue and Yellow bunch intensity. With Eq. (4) the luminosity lifetime can be calculated as

$$
\frac{1}{\tau_{\mathcal{L}}}=\frac{1}{\tau_{N_{b}, B}}+\frac{1}{\tau_{N_{b}, Y}}+\frac{1}{\tau_{\epsilon_{n}}} .
$$

where $\tau_{N_{b}, B}$ and $\tau_{N_{b}, Y}$ are the beam lifetimes for the Blue and Yellow beam respectively.

\section{Lifetime observations in Run-5}

We will report on observations of beam and luminosity lifetimes, from which emittance growth times can be calculated. We also show the observed bunch length evolution during stores.

\section{$2.1 \quad$ Beam lifetimes}

The time dependent proton intensities $N_{b}(t)$ of all physics stores (see Fig. 1 ) were fitted with either a single or double exponential function

$$
N_{b}(t)=A_{1} e^{-t / \tau_{N_{b}, 1}}+A_{2} e^{-t / \tau_{N_{b}, 2}} .
$$

In Fig. 2 the fitted Blue and Yellow beam lifetimes are shown. The left hand side show the fast decaying component, centered at $0.38 \mathrm{~h}$ and $0.41 \mathrm{~h}$ for the Blue and Yellow beam respectively. Both distributions are relatively narrow. The right hand side of Fig. 2 shows the slow decaying part. These distributions are much wider. A large number of Yellow stores have slow beam decay times of less than $50 \mathrm{~h}$. The average slow decay times for the Blue and Yellow beam are $90 \mathrm{~h}$ and $57 \mathrm{~h}$ respectively. The beam lifetime of approximately one third of all physics stores could be well fit to a single exponential decay function, typically for both the Blue and Yellow beams. Most of these stores are in the early part of the run when the performance was still improving.
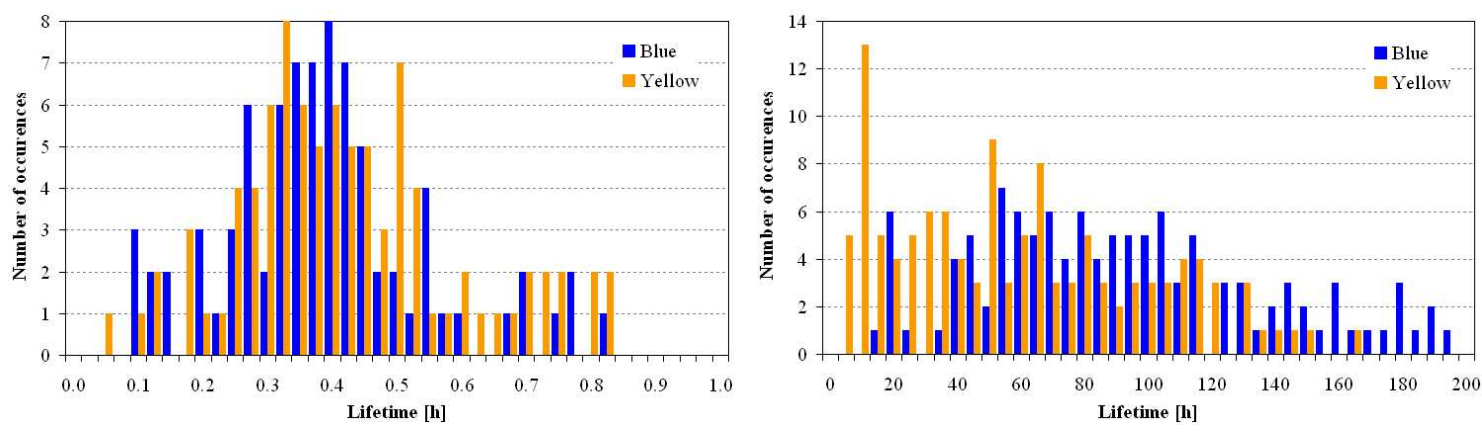

Figure 2: Beam lifetimes were fitted with a double-exponential function $N_{b}(t)=A_{1} e^{-t / \tau_{N_{b}, 1}}+A_{2} e^{-t / \tau_{N_{b}, 2}}$. Histograms of the fast (left) and slow (right) beam lifetime components for the polarized proton physics stores of Run-5. 


\subsection{Luminosity lifetime}

Like the beam lifetimes, the time dependent luminosities $\mathcal{L}(t)$ of all physics stores (see Fig. 1) were fitted with either a single or double exponential function

$$
\mathcal{L}(t)=A_{1} e^{-t / \tau_{\mathcal{L}, 1}}+A_{2} e^{-t / \tau_{\mathcal{L}, 2}} .
$$

All fits start when the luminosity signal is free from background contamination. This can take up to half an hour after the beams were brought into collision. Background conditions were not reproducible either, and backgrounds at STAR were thought to correlate with horizontal orbit bumps in IP6. The STAR experiment had no shielding installed in Run-5.

Fig. 3 shows the fast (left) and slow (right) luminosity lifetime distributions. The fast and slow components of the luminosity decay have average values of $0.3 \mathrm{~h}$ and $11 \mathrm{~h}$ respectively. The fast decay time is approximately the same as the fast beam decay time. From the average beam and luminosity lifetimes, the average emittance growth time can be calculated with Eq.(5), shown in Tab. 2.
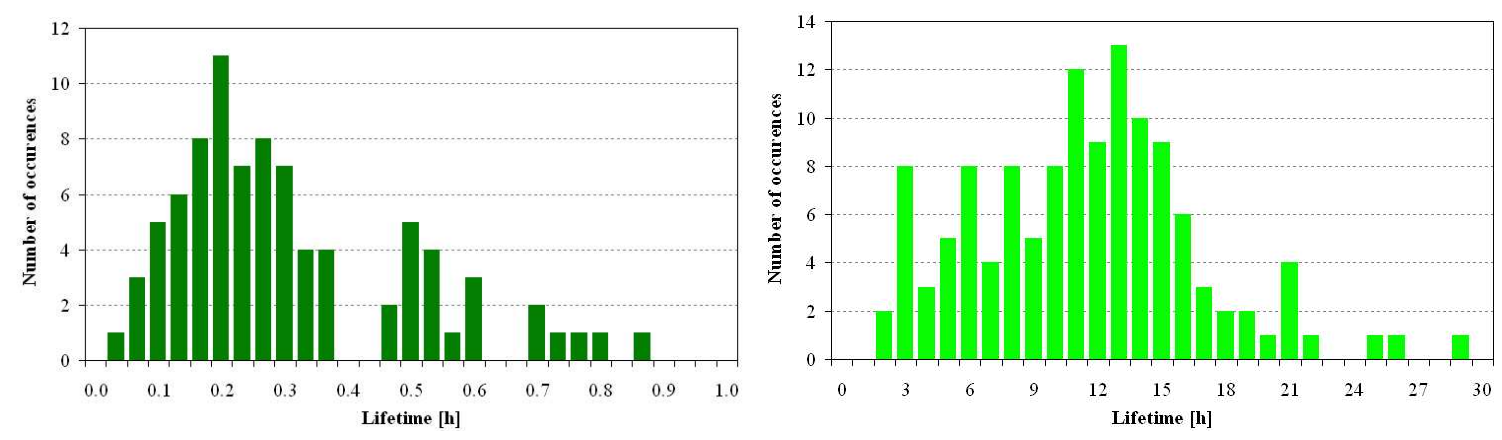

Figure 3: Luminosity lifetimes were fitted with a double-exponential function $\mathcal{L}(t)=A_{1} e^{-t / \tau_{\mathcal{L}, 1}}+$ $A_{2} e^{-t / \tau_{\mathcal{L}, 2}}$. Histograms of the fast (left) and slow (right) luminosity lifetimes components for the polarized proton physics stores of Run-5.

\subsection{Bunch length evolution}

The rf voltage in store is $300 \mathrm{kV}$. The bunch length is measured with a wall current monitor. The FWHM of all bunches is averaged, and the time evolution of the bunch length is fitted to a $3^{\text {rd }}$ order polynomial. The rms bunch length is calculated assuming a Gaussian bunch shape.

The bunch length evolution of the Run-5 stores is shown in Fig. 4. The average Blue bunch length at the beginning of a store is $0.76 \mathrm{~m}$. It increases by $20 \%$ to $0.91 \mathrm{~m}$ after $3 \mathrm{~h}$. The average Yellow bunch length at the beginning of the store is $0.88 \mathrm{~m}, 16 \%$ larger than the Blue one. The average Yellow bunch length increases by $11 \%$ to $0.98 \mathrm{~m}$ after $3 \mathrm{~h}$. A systematically larger Yellow bunch length has also been observed with $\mathrm{Cu}$ beams. The systematically larger bunch length could contribute to the reduced beam lifetime, and observed reduced momentum aperture. The average longitudinal emittance growth time at the beginning of the store is $7.6 \mathrm{~h}$. 
The right hand side of Fig. 4 shows the Blue and Yellow bunch length just before and after the energy ramp starts. Before the ramp there is no difference between Blue and Yellow. After the ramp has started, the Yellow bunches lengthen. This could be due to the fact that the Yellow rf frequency is the slave to the Blue rf frequency. It is not clear why the Blue bunch length distribution shifts to smaller values at the beginning of the ramp.
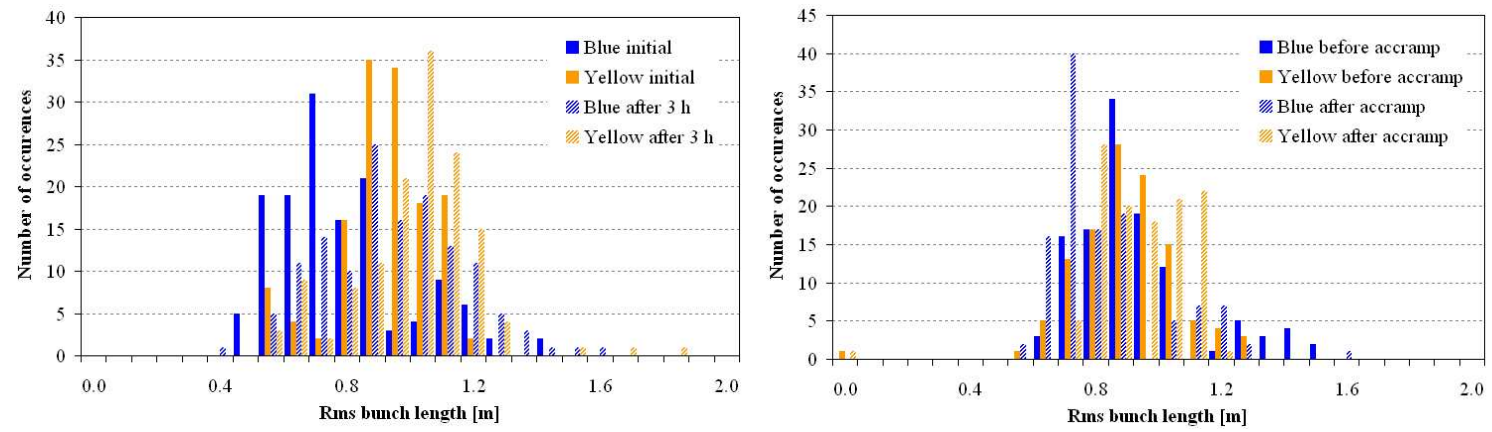

Figure 4: Left: Rms bunch length at the beginning of stores, and $3 \mathrm{~h}$ later. Right: Rms bunch length just before and after the start of the energy ramp.

\subsection{Further observations}

When bringing the beams into collision, both beams show increased losses, but in most cases the Yellow beam suffered more than the Blue beam (Fig. 5). The Yellow beam loss rate varied widely from store to store.

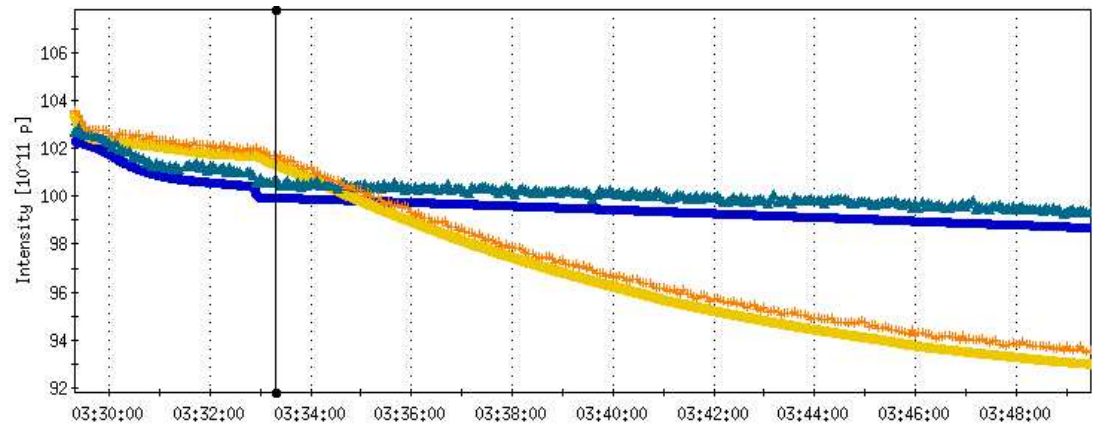

Figure 5: Effect of the beam-beam interaction on the beam lifetime at the beginning of the store. The vertical line marks the time when beams are brought into collision. In most cases the Yellow beam lifetime deteriorated visibly.

Only a weak correlation to the local orbits in the interaction regions can be established. Fig. 6 shows the beam intensity as well as the horizontal and vertical angle bumps in IP6 from April 30 to May 4, 2005. The Yellow beam lifetime fluctuates over a wide range, while the orbit angle through the IP changes. 

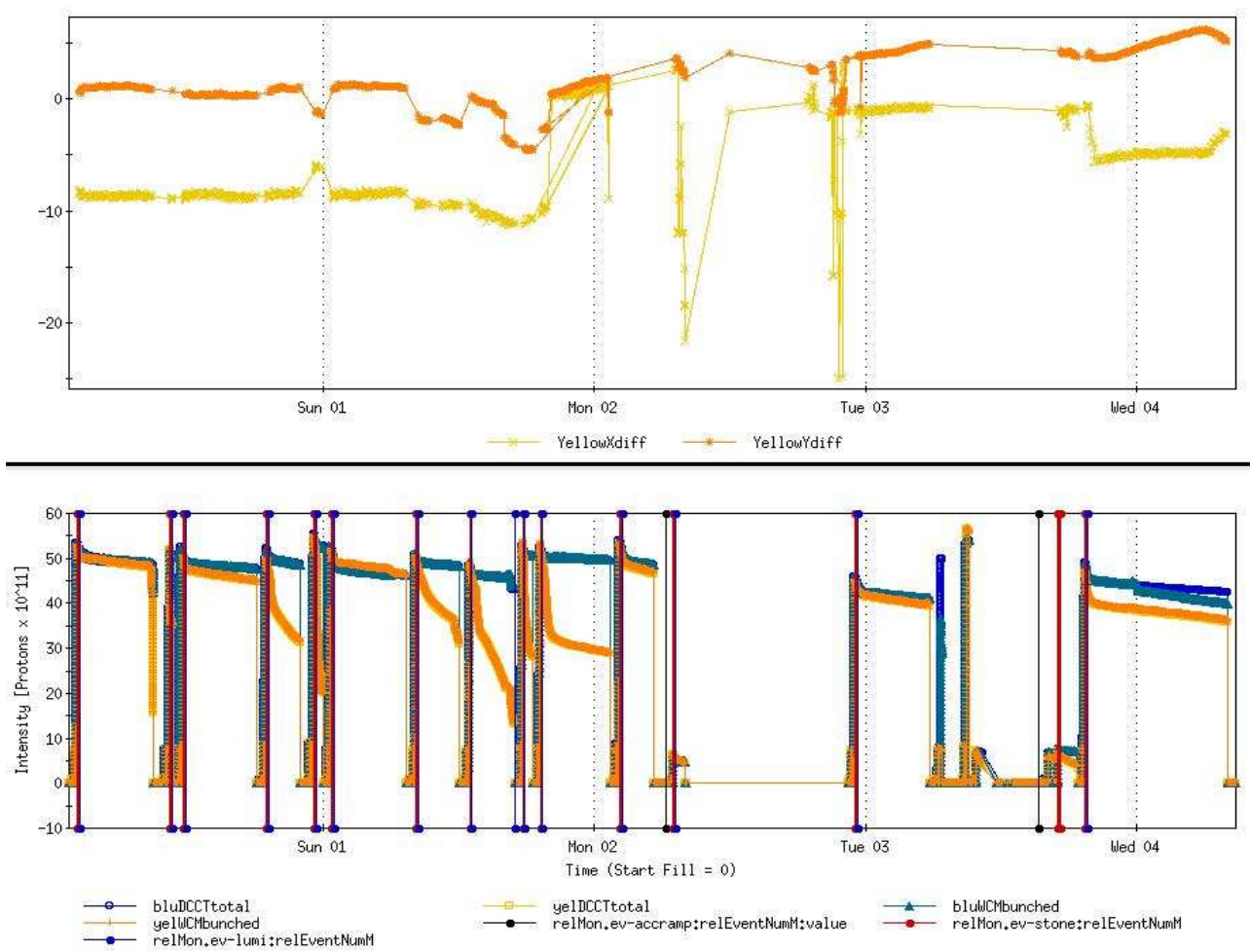

Figure 6: Blue and Yellow beam intensities (bottom), and Yellow orbit angles in IP6. YellowXdiff and YellowYdiff denote the difference (in $\mathrm{mm}$ ) between the Yellow triplet BPMs. They are a measure of the orbit angle through the IP.

The RHIC orbit undergoes diurnal vertical oscillations [3]. The vertical angle in IP6 was found to be a good representative of these orbit movements, and the IR4 triplets were identified as the main source [4]. The angle excursions at IP6 reached a maximum around $5 \mathrm{am}$ and $5 \mathrm{pm}$ every day. Fig. 7 shows the fast decaying part of the luminosity as a function of the time of the day. When the Yellow vertical orbit angle is near a maximum, the fraction of the luminosity that is decaying fast is increased. As similar plot for copper stores does not show any dependence on the time of the day. The total beam-beam induced tune spread with copper beams ( 4 head-on collisions with $\xi \approx 0.0025 / \mathrm{IP}$ ) is close to the total beam-beam induced tune spread of proton beams ( 3 head-on collisions with $\xi \approx 0.0040 / \mathrm{IP})$. However, proton beams are about $50 \%$ larger in the triplet due to the same $\beta^{*}$ at lower rigidity, and are thus more susceptible to nonlinear magnetic field errors in these locations.

\section{Beam loss and emittance growth estimates}

We estimate the beam loss due to luminosity and rest gas inelastic scattering, and the emittance growth due to intrabeam scattering, rest gas elastic scattering, and elastic 


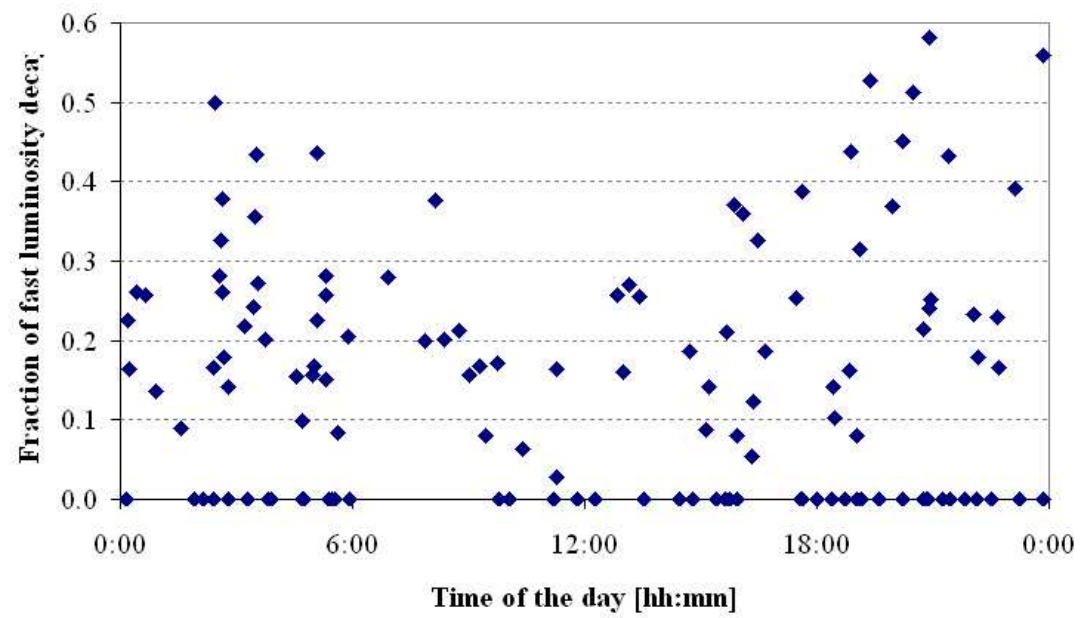

Figure 7: Fraction of the fast luminosity decay as a function of the time of the day.

beam-beam scattering. Estimates are made for a Run-5 reference case, with parameters close to those achieved in the best stores, for the Enhanced Luminosity parameters at $100 \mathrm{GeV}$ and $250 \mathrm{GeV}$ beam energy, and for the RHIC II parameters at $250 \mathrm{GeV}$ (Tab. 2). All calculations are for the beginning of the store, when the lifetimes and emittance growth times are shortest.

\subsection{Beam loss from luminosity}

In Ref. [2] a total cross sections $\sigma_{t o t}$ and elastic cross sections $\sigma_{e l}$ are given for protonproton collisions over a large range of energies. For beam energies of $100 \mathrm{GeV}$ and $250 \mathrm{GeV}$ respectively, the numbers shown in Tab. 2 can be extracted.

To make an estimate for the luminous beam and luminosity decay we assume that all particle interactions due to the total proton-proton cross section lead to the loss of both protons involved, and that there is no emittance growth. The particle loss per beam is then

$$
n_{I P} N \frac{d N_{b}}{d t}=-\mathcal{L}(t) \sigma_{t o t}
$$

where $n_{I P}$ is the number of interaction points, $N$ the number of colliding bunches, and $N_{b}$ the bunch intensity. With the luminosity given by Eq. (4) the solution for $N_{b}(t)$ and $\mathcal{L}(t)$ are

$$
N_{b}(t)=\frac{N_{b}(0)}{1+t / \tau} \quad \text { and } \quad \mathcal{L}(t)=\frac{\mathcal{L}(0)}{(1+t / \tau)^{2}}
$$

with

$$
\tau=\frac{N N_{b}(0)}{n_{I P} \mathcal{L}(0) \sigma_{\text {tot }}}=\frac{2 \pi}{3(\beta \gamma)} \frac{\epsilon_{n} \beta^{*}}{n_{I P} f_{\text {rev }} N_{b}(0) \sigma_{\text {tot }}}
$$

At $t=0$ we have $\tau_{N_{b}}=\tau$. Calculated lifetimes are in Tab. 2. These are much larger than the observed beam lifetimes in Run- 5 . 
Table 2: Beam loss and emittance growth calculations for proton-proton collisions in RHIC. All parameters are for the beginning of store. The Run- 5 parameters are close to those achieved in the best stores. The Enhanced RHIC I, and RHIC II parameters are design goals.

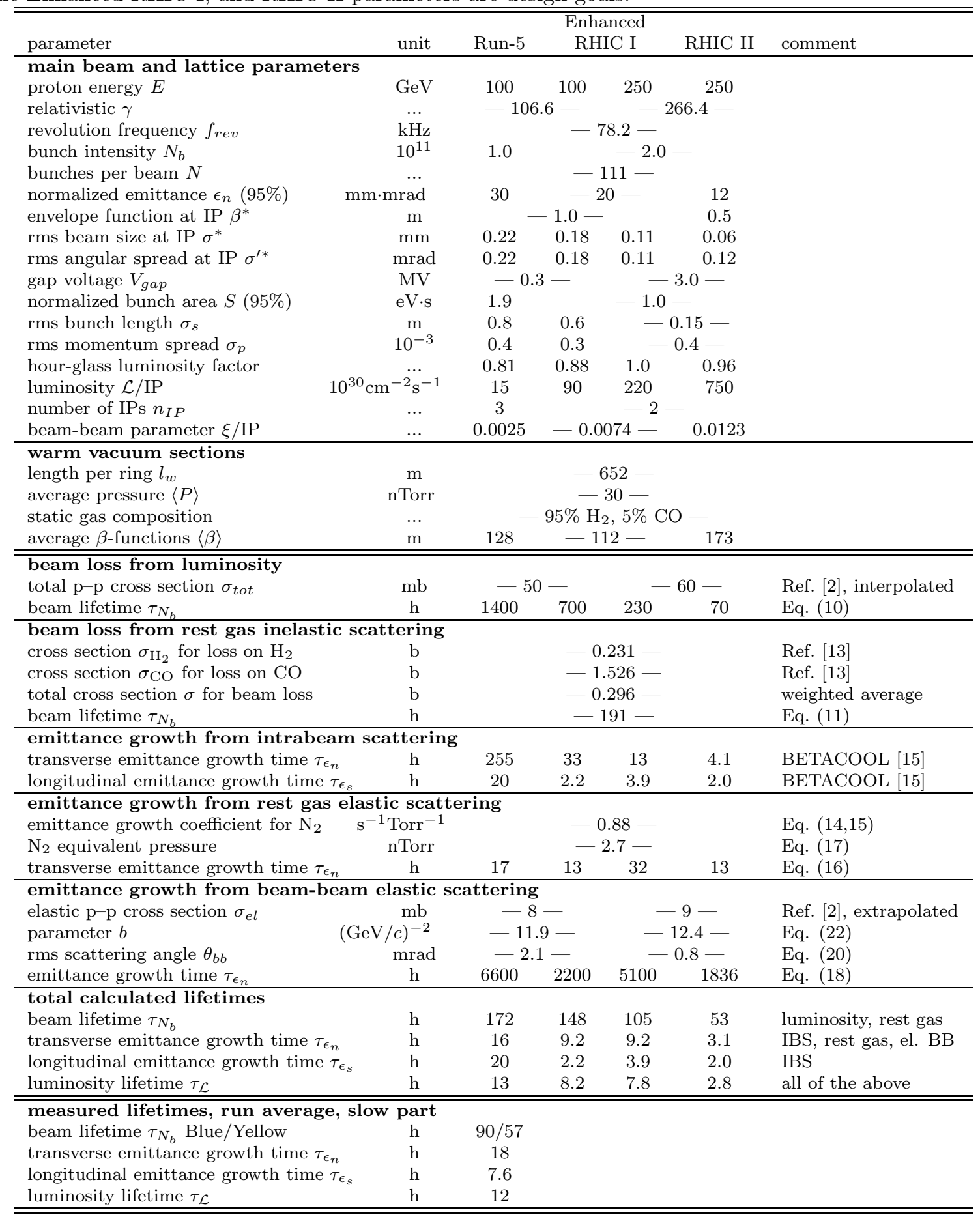




\subsection{Beam loss from rest gas inelastic scattering}

We consider only the warm sections of the ring, which constitutes $17 \%$ of the circumference (Tab. 3). The gas density in the cold parts of the ring is much lower due to the cryo pumping of the $4 \mathrm{~K}$ cold beam pipe walls. To calculate the beam loss and emittance growth from rest gas scattering, the average pressure is needed. The pressure given in Tab. 2 is typical, although we note that the pressure can vary by orders of magnitude from location to location, and with dynamic effects, of which electron cloud induced desorption is the most important one [6].

Table 3: Locations and average $\beta$-functions of the RHIC warm sections. $\beta^{*}=1.0 \mathrm{~m}$ and $\beta^{*}=0.5 \mathrm{~m}$ is only possible in IR6 and IR8. These interaction regions also have spin rotators. $\beta$-functions are given for one side of the IR. Due to the anti-symmetry of the IR optics, $\beta_{x}$ and $\beta_{y}$ are exchanged on the other side.

\begin{tabular}{lccccc}
\hline \hline section & $\begin{array}{c}\text { sections } \\
\text { per ring }\end{array}$ & $\begin{array}{c}\text { location } \\
\text { from IP } \\
{[\mathrm{m}]}\end{array}$ & $\begin{array}{c}\text { section } \\
\text { length } \\
{[\mathrm{m}]}\end{array}$ & $\begin{array}{c}\left\langle\beta_{x, y}\right\rangle \\
{[\mathrm{m}]}\end{array}$ & $\begin{array}{c}\left\langle\beta_{y, x}\right\rangle \\
{[\mathrm{m}]}\end{array}$ \\
\hline IP-D0 & 12 & $0-20$ & 20 & 30 & 30 \\
Q3-rotator (IR6 \& IR8) & 4 & $38-60$ & 22 & 21 & 85 \\
Q3-Q4 & 8 & $38-76$ & 38 & 23 & 63 \\
Q7-Q8 (injection) & 1 & $114-126$ & 12 & 26 & 28 \\
Q9-D9 (injection) & 1 & $142-150$ & 8 & 16 & 35 \\
\hline & & & & $-\beta^{*}=1.0 \mathrm{~m}-$ \\
IP-D0 & 12 & $0-12$ & 20 & 200 & 200 \\
Q3-rotator (IR6 \& IR8) & 4 & $38-60$ & 22 & 197 & 724 \\
Q7-Q8 (injection) & 1 & $114-126$ & 12 & 24 & 24 \\
Q9-D9 (injection) & 1 & $142-150$ & 8 & 16 & 35 \\
\hline & & & & $-\beta^{*}=0.5 \mathrm{~m}-$ \\
IP-D0 & 12 & $0-20$ & 20 & 400 & 400 \\
Q3-rotator (IR6 \& IR8) & 4 & $38-60$ & 22 & 322 & 1160 \\
Q7-Q8 (injection) & 1 & $114-126$ & 12 & 26 & 25 \\
Q9-D9 (injection) & 1 & $142-150$ & 8 & 17 & 18 \\
\hline \hline
\end{tabular}

The beam lifetime due to rest gas inelastic scattering of protons on molecular nitrogen $N_{2}$ at $300 \mathrm{~K}$ is $[9]$

$$
\frac{1}{\tau_{N_{b}}}=-\frac{1}{N_{b}} \frac{d N_{b}}{d t} \approx 800 \mathrm{~s}^{-1} \operatorname{Torr}^{-1} \frac{\beta}{C} \int_{0}^{C} P(s) d s .
$$

For a gas mixture the nitrogen equivalent pressure can be calculated as $[9]$

$$
P_{N_{2} e q i v}^{n u c l}=0.0861 \sum_{i} P_{i} \sum_{j} k_{i j} A_{i j}^{2 / 3}
$$

where $P_{i}$ is the partial pressure of gas molecules $i, k_{i j}$ are the number of species $j$ in the gas molecule $i$, and $A_{i j}$ the mass number of species $j$ in molecule $i$. 


\subsection{Emittance growth from intrabeam scattering}

The emittance growth times due to intrabeam scattering of Gaussian beam profiles in all three dimensions can be obtained as [18]

$$
\frac{1}{\tau_{x, y, s}}=\frac{Z^{4}}{A^{2}} C_{x, y, x} \frac{N_{b}}{\gamma \epsilon_{x} \epsilon_{y} \epsilon_{s}}
$$

where $Z$ and $A$ are the charge and mass numbers of the particles in the beam, and the coefficients $C_{x, y, x}$ a function of the lattice. Calculations of the emittance growth times were done with the code BETACOOL [15] by A. Fedotov [16]. A transversely fully coupled lattice was assumed so that the emittance growth times for the horizontal and vertical plane are equal. Calculations with BETACOOL were in good agreement with dedicated measurements of copper beams in Run-5 [19].

The calculated emittance growth times for Run-5 are significantly larger than the observed ones. However, for the Enhanced RHIC I, and the RHIC II parameters intrabeam scattering growth times will be significant. Note that we have assumed the use of the storage rf system for these cases.

\subsection{Emittance growth from rest gas elastic scattering}

We consider again only the warm sections of RHIC. With the emittance definition Eq. (1) the emittance growth time due to rest gas elastic scattering is $[5,9]$

$$
\frac{1}{\tau_{\epsilon_{n}}}=\frac{1}{\epsilon_{n}} \frac{d \epsilon_{n}}{d t}=\frac{6(\beta \gamma)}{\epsilon_{n}} \times \frac{1}{C} \int_{0}^{C} \beta(s) \theta_{r g}^{2}(s) d s
$$

where $C$ is the circumference and $\theta_{r g}$ the rms scattering angle. For protons, scattering on species with atomic number $Z$ and density $n$, the scattering angle is [9]

$$
\theta_{r g}^{2}(s)=\frac{4 \pi r_{p}^{2} c}{\beta^{3} \gamma^{2}} n Z(Z+1) \ln \left(183 Z^{-1 / 3}\right)
$$

where $r_{p}$ is the classical proton radius and $c$ the speed of light. For molecular nitrogen $\mathrm{N}_{2}$ at $300 \mathrm{~K}$ one has

$$
\frac{1}{\tau_{\epsilon_{n}}} \approx 0.88 \mathrm{~s}^{-1} \operatorname{Torr}^{-1} \frac{l_{w}}{C} \frac{\langle\beta P\rangle}{\epsilon_{n} \gamma}
$$

where $l_{w}$ is the length of the warm sections. For other rest gas species the $\mathrm{N}_{2}$ equivalent pressure can be calculated according to Ref. [9] as

$$
P_{N_{2} \text { equiv }}^{\text {elast }}=\frac{2 \times 10^{-3}}{P} \sum_{i} P_{i} \sum_{j} k_{i j} Z_{i j}\left(Z_{i j}+1\right) \ln \left(183 Z_{i j}^{-1 / 3}\right),
$$

where $P_{i}$ is the partial pressure of gas molecules $i$, and $k_{i j}$ the number of species $j$ in the gas molecule $i$. Average $\beta$-functions of the warm sections are displayed in Tab. 3 . Calculated emittance growth times in Tab. 2 show values comparable to the measured values. We note again that the dynamic pressure at the beginning of stores can be higher than the value given in Tab. 2, and the emittance growth times correspondingly shorter. 


\subsection{Emittance growth from beam-beam elastic scattering}

The emittance growth due to beam-beam elastic scattering at a single IP is $[5,9]$

$$
\frac{1}{\tau_{\epsilon_{n}}}=\frac{1}{\epsilon_{n}} \frac{d \epsilon_{n}}{d t}=\frac{9}{2} \gamma n_{I P} \frac{f_{r e v} N_{b}}{\epsilon_{n}^{2}} \sigma_{e l} \theta_{b b}^{2}
$$

where $\theta_{b b}$ is the rms scattering angle. At high energies, the differential cross section is well described by a simple exponent $[9,10]$

$$
\frac{d \sigma_{e l}}{d t} \sim e^{-b|t|}=\exp \left\{-\frac{\theta^{2}}{2 \theta_{b b}^{2}}\right\}
$$

where $t \approx-p^{2} \theta^{2}$ is the square of the 4 -momentum, and $p$ the proton momentum. The rms scattering angle is then

$$
\theta_{b b}=\frac{1}{p \sqrt{2 b}} .
$$

The parameter $b$ can be calculated in the momentum range from $5 \mathrm{GeV} / c$ to many $\mathrm{TeV} / c$ according to $[11,12]$

$$
b(p)=b_{0}+b_{1} \sqrt{\frac{p_{0}}{p}}+b_{2} \ln \left(\frac{p}{p_{0}}\right)
$$

with fitted parameters $b_{0}, b_{1}$, and $b_{2}$ from Ref. [11]:

$$
\begin{aligned}
& p_{0}=1(\mathrm{GeV} / c) \\
& b_{0}=+11.13 \pm 0.22(\mathrm{GeV} / c)^{-2} \\
& b_{1}=-6.21 \pm 0.53(\mathrm{GeV} / c)^{-2} \\
& b_{2}=+0.30 \pm 0.04(\mathrm{GeV} / c)^{-2} .
\end{aligned}
$$

Calculated values for $b, \theta_{b b}$ and the emittance growth rate are shown in Tab. 2. Note that the rms scattering angles are much larger than the rms angular spread of the beam. Almost all scattered particles will therefore be lost at the collimators. The lost particles are accounted for in the beam loss from luminosity in Sec. 3.1, that uses the p-p total cross section $\sigma_{\text {tot }}$. The emittance growth due to this effect can be neglected.

\section{Summary}

The Run-5 beam and luminosity lifetimes show large variations. Both the beam intensity and the luminosity typically had a fast and slow decaying component. The fast decaying beam and luminosity component had average lifetime of approximately $0.5 \mathrm{~h}$. The slow decaying component of the beam lifetime had an average lifetime of $90 \mathrm{~h}$ and $57 \mathrm{~h}$ in the Blue and Yellow ring respectively. The reason for the large lifetime variations could not be identified conclusively, although some correlation with orbit variations can be found. The slow decaying luminosity component had an average lifetime of $11 \mathrm{~h}$. 
Calculations of luminosity lifetimes that include beam losses from luminosity and rest gas scattering, and emittance growth from rest gas scattering, intrabeam scattering, and beam-beam elastic scattering agree reasonably well with the measured slow luminosity lifetime component for the largest dynamic pressures. However, not included in the luminosity lifetime calculations are effects from the nonlinear magnetic field errors of the triplets and the beam-beam interactions. The assumed dynamic pressure also has a large error due to its variation by orders of magnitude around the ring, and its time dependence. The luminosity lifetime calculations should therefore only be seen as preliminary. The calculations for the planned proton upgrades show a significant intra-beam scattering effect for the RHIC II conditions.

\section{Acknowledgments}

For help and discussions the authors are thankful to V. Balbekov, M. Brennan, A. Fedotov, N. Mokhov, T. Roser, F. Pilat, V. Ptitsyn, D. Trbojevic, T. Sen, and the members of the RHIC operations teams.

\section{References}

[1] W. Fischer, "RHIC run overview", http://www.agsrhichome.bnl.gov/RHIC/Runs/ (2005).

[2] S. Eidelman et al., Phys. Lett. B 592, 1. Fig. 40.11 (2004) and 2005 partial update for the 2006 edition available on the PDG WWW pages (URL: http://pdg.lbl.gov/); Data courtesy of COMPASS group, IHEP, Protvino (August 2005).

[3] V. Ptitsyn, "Orbit mysteries: clues and cures", RHIC Retreat, June 2005, http://www.c-ad.bnl.gov/RHIC/Retreat2005/ (2005).

[4] D.Trbojevic, D. Bruno, V. Ptitsyn, P. Thieberger, private communication (2005).

[5] M. Syphers, "Emittance dilution effects [1]", in "Handbook of accelerator physics and engineering", World Scientific, (1998).

[6] D. Weiss, H.C. Hseuh, "Vacuum and NEG pipes", RHIC Retreat, June 2005, http://www.c-ad.bnl.gov/RHIC/Retreat2005/ (2005).

[7] F. Pilat, Y. Luo, N. Malitsky, and V. Ptitsyn, "Beam-based nonlinear optics corrections in colliders", proceedings of the 2005 Particle Accelerator Conference, Knoxville, TN (2005).

[8] F. Pilat, private communication (2006).

[9] N.V. Mokhov and V.I. Balbekov, "Beam and luminosity lifetime", in "Handbook of accelerator physics and engineering", World Scientific, (1998). 
[10] R. Rubinstein, "Total, elastic and diffractive cross sections at high energies", FERMILAB-Conf-90/160-E [E-710] (1990).

[11] J.P. Burq, M. Chemarin, M. Chevallier, A.S. Denisov, C. Doré, T. Ekelöf, J. Fay, P. Grafström, L. Gustafsson, E. Hagberg, B. Ille, A.P. Kashchuk, G.A. Korolev, A.V. Kulikov, S. Kullander, M. Lambert, J.P. Martin, S. Maury, M. Querrou, V.A. Verbeken and A.A. Voobyov, "Soft $\pi^{-}$p and pp elastic scattering in the energy range 30 to 345 GeV", Nucl. Phys. B 217 pp. 285-335 (1982).

[12] N. Mokhov, private communication (2006).

[13] D. Trbojevic, W. Fischer, H.-C. Hseuh, W.W. MacKay, and S.Y. Zhang, "Comparison between the predictions and measurements for the beam gas interactions during the last gold and proton runs in RHIC", proceedings of the 2002 European Particle Accelerator Conference, Paris, BNL-69006 (2002).

[14] D. Trbojevic, "The beam lifetime and emittance growth in RHIC under normal operating conditions and with a hydrogen gas jet", BNL RHIC/AP/136 (1997).

[15] I. Meshkov, A. Sidorin, A. Smirnov, E. Syresin, and G. Trubnikov, BETACOOL program, http://Lepta.jinr.ru/betacool.htm (1996-2006).

[16] A. Fedotov, private communication (2006).

[17] B. Jeanneret and F. Zimmermann, "What is an acceptable vacuum pressure in the LHC arcs?", proceedings of the First CARE-HHH-APD Workshop on Beam Dynamics in Future Hadron Colliders and Rapidly Cycling High-Intensity Synchrotrons (HHH-2004), CERN, Geneva, Switzerland, CERN-2005-006 (2005).

[18] A. Piwinski, "Touschek effect and intrabeam scattering", in "Handbook of accelerator physics and engineering", World Scientific, (1998).

[19] A. Fedotov, R. Connolly, W. Fischer, V. Litvinenko, S. Tepikian, J. Wei, "IBS studies in RHIC (2004-2005)", 2005 RHIC Accelerator Physics Experiements Workshop, BNL, www.c-ad.bnl.gov/APEX/ (2005). 